\title{
The prognosis factors among breast cancer patients with extensive axillary lymph node metastasis
}

\author{
Jae-Myung Kim', Ju-Yeon Kim', Eun Jung Jung², Seung Jin Kwag', Ji-Ho Park', Taejin Park², Sang-Ho Jeong², Chi-Young Jeong', \\ Young-Tae Ju', Young-Joon Lee', Soon-Chan Hong' ${ }^{1}$ \\ 'Department of Surgery, Gyeongsang National University Hospital, Gyeongsang National University School of Medicine, Jinju; \\ ${ }^{2}$ Department of Surgery, Gyeongsang National University Changwon Hospital, Gyeongsang National University School of Medicine, Changwon, Korea
}

Purpose: This study was designed to retrospectively identify prognostic factors of survival among breast cancer patients with 10 or more metastatic lymph nodes (LNs).

Methods: The study included 58 patients with 10 or more metastatic LNs who received standard treatment from January 2005 to December 2015. To identify the prognostic factors, we analyzed the difference of disease-free survival (DFS) according to clinicopathologic factors.

Results: The 5-year DFS and overall survival rates in all patients were 55\% and 69\%, respectively. Tumor size, number of metastatic LNs and ratio of metastatic to total LNs were associated with poorer prognosis. DFS was significantly poorer in patients with $>15$ than $\leq 15$ metastatic LNs (hazard ratio [HR], 4.60; 95\% confidence interval [Cl], 1.38-15.32) and with $\mathrm{LN}$ ratio $>0.64$ than $\leq 0.64(\mathrm{HR}, 26.13 ; 95 \% \mathrm{Cl}, 3.16-$ 215.80) A scoring system based on these factors was significantly prognostic of survival outcomes.

Conclusion: This study identified factors of survival in breast cancer patients with extensive LN metastasis. Patients with unfavorable factors may require modified management to improve their clinical outcomes.

Keywords: Breast neoplasms, Prognosis, Lymphatic metastasis

\section{INTRODUCTION}

Invasive breast cancer is one of the most common malignancies in women. The most common sites of metastasis are the axillary lymph nodes (LNs). Moreover, the number of metastasis-positive LNs is considered the most powerful predictor of patient prognosis.

Despite screening tests, approximately $8 \%$ to $30 \%$ of newly diagnosed breast cancers are at an advanced stage, with extensive LN metastasis [1,2]. Although the development of multimodal treatments has improved survival outcomes in breast cancer patients, outcomes remain unsatisfactory in patients with multiple axillary LN metastases [3]. Older treatment regimens resulted in 5-year

Received: Nov 2, 2017 Accepted: May 15, 2018

Correspondence to: Eun Jung Jung

Department of Surgery, Gyeongsang National University Changwon

Hospital, Gyeongsang National University School of Medicine,

11 Samjeongja-ro, Seongsan-gu, Changwon 51472, Korea

Tel: +82-55-750-8732, Fax: +82-55-757-5442

E-mail: drjej@gnu.ac.kr

Copyright $($ Korean Society of Surgical Oncology

This is an Open Access article distributed under the terms of the Creative Commons Attribution Non-Commercial License (http://creativecommons.org/licenses/by-nc/4.0) which permits unrestricted non-commercial use, distribution, and reproduction in any medium, provided the original work is properly cited. overall survival (OS) rates of approximately 40\% [4,5], more recent advanced treatment modalities have resulted in 5-year OS rates of 66\% and $81 \%$ [6]. Despite these improvements, however, factors prognostic of survival in patients with extensive LN metastasis remain unclear.

This study was therefore designed to identify clinical factors prognostic of survival in patients with extensive LN metastasis.

\section{METHODS}

Node dissection was confined to levels I and II axillary LNs in patients not suspected of having level III, supraclavicular, and/or internal mammary metastatic LNs. Adjuvant radiation therapy was administered to all pathologic N3 patients who underwent breast-conserving surgery or mastectomy. Patients who refused adjuvant chemotherapy, radiation and/or hormonal therapy were excluded from this study.

Extensive axillary nodal metastasis was defined as $\geq 10$ axillary nodes on pathology reports (pN3a). LN ratio was defined as the number of involved nodes divided by the number of LNs examined.

Disease-specific events included locoregional recurrence, dis- 
tant metastasis and contralateral breast cancer. Disease-free survival (DFS) was defined as the time from curative surgery to the date of the first disease-specific events or last follow-up. OS was defined as the time from curative surgery to the date of death from breast cancer or last follow-up. Patient survival outcomes were assessed by the Kaplan-Meier method and compared by the log-rank test. The Cox regression model was utilized to identify significant independent factors associated with distant metastasis. Significance was defined as $\mathrm{P}<0.05$. All statistical analyses were performed using SPSS version 19.0 (IBM Corp., Armonk, NY, USA).

\section{RESULTS}

Fifty-eight patients were included in this study; their clinical and histopathological characteristics are listed in Table 1. The mean age of all patients was 52 years (range, 29-76 years), and 24 patients (41.4\%) were over 50 years of age. Fifteen patients (25.9\%) under-

Table 1. Clinical characteristics of study patients

\begin{tabular}{lc}
\hline Characteristics & Value $(\mathrm{n}=58)$ \\
\hline Age $(\mathrm{yr})$ & $51.67 \pm 10.93$ \\
Tumor size $(\mathrm{cm})$ & $3.11 \pm 1.77$ \\
$\leq 2$ & $18(31.0)$ \\
$2-5$ & $33(56.9)$ \\
$>5$ & $7(12.1)$ \\
Estrogen receptor & \\
Positive & $29(50.0)$ \\
Negative & $29(50.0)$ \\
Progesterone receptor & \\
Positive & $29(50.0)$ \\
Negative & $29(50.0)$ \\
HER2 & \\
Positive & $18(31.0)$ \\
Negative & $37(63.8)$ \\
Unknown & $3(5.2)$ \\
Grade & \\
I and II & $24(41.4)$ \\
III & $31(53.4)$ \\
Unknown & $3(5.2)$ \\
Lymphovascular invasion & \\
Present & $19(32.8)$ \\
Absent & $39(67.2)$ \\
Operation type & \\
Mastectomy & $43(74.1)$ \\
Conservation & $15(25.9)$ \\
LN ratio) & $0.72 \pm 0.20(0.31-1.00)$ \\
No. of metastatic LN & $20.81 \pm 13.58$ \\
\hline & \\
\hline & \\
\hline &
\end{tabular}

Values are presented as mean \pm standard deviation or number (\%). HER2, human epidermal growth factor receptor 2; LN, lymph node.

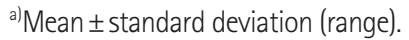

went breast-conserving surgery. Most patients, except those with poor performance status, were treated with taxane-based adjuvant chemotherapy. Twelve patients $(20.7 \%)$ received anthracycline without taxane-based chemotherapy. The median number of LNs dissected was 24 (range, 11-62), and the median number of metastatic LNs was 17 (range, 10-61).

After a mean follow-up of 46.3 months (range, 6-112 months), 20 patients (34.5\%) showed evidence of recurrence, including 17 (29.3\%) with distant metastases. The median time between surgery and disease recurrence was 29.0 months, and seven patients (12.1\%) died of breast cancer-related causes. The 5-year DFS and OS rates in all 58 patients were $55 \%$ and $69 \%$, respectively.

Factors prognostic of DFS are listed in Table 2. Tumor size $>5$ $\mathrm{cm}$ was associated with poorer prognosis, whereas the subtype of breast cancer was not associated with DFS. As continuous variables, LN ratio and number of metastatic LNs were associated with DFS. The cutoffs of LN ratio and number of metastatic LNs were determined by receiver operating characteristic (ROC) curves, with the areas under the ROC curves being 0.813 (95\% confidence interval [CI], 0.70-0.93) and 0.709 (95\% CI, 0.57-0.84), respec-

Table 2. Characteristics and DFS

\begin{tabular}{|c|c|c|c|}
\hline Characteristics & DFS $(\mathrm{mo})^{\mathrm{a})}$ & $\begin{array}{l}\text { Log-rank } \\
\text { P-value }\end{array}$ & $\begin{array}{c}\text { Univariate hazard } \\
\text { ratio }(95 \% \mathrm{Cl})\end{array}$ \\
\hline Age (yr) & & 0.095 & \\
\hline$\leq 50$ & $88.16 \pm 8.78$ & & Reference \\
\hline$>50$ & $53.96 \pm 10.15$ & & $2.10(0.86-5.12)$ \\
\hline Tumor size $(\mathrm{cm})$ & & 0.044 & \\
\hline$\leq 5$ & $84.62 \pm 7.80$ & & Reference \\
\hline$>5$ & $35.00 \pm 8.39$ & & $2.38(1.05-14.42)$ \\
\hline Subtype & & 0.330 & \\
\hline HR+/HER2- & $68.74 \pm 7.41$ & & Reference \\
\hline HR+/HER2+ & $36.40 \pm 8.66$ & & $1.67(0.23-11.93)$ \\
\hline HR-/HER2+ & $90.06 \pm 15.90$ & & $0.75(0.16-3.46)$ \\
\hline HR-/HER2- & $43.94 \pm 13.15$ & & $5.00(1.00-25.02)$ \\
\hline Grade & & 0.740 & \\
\hline | and || & $68.32 \pm 11.57$ & & Reference \\
\hline III & $80.47 \pm 9.48$ & & $0.86(0.34-2.15)$ \\
\hline Lymphovascular invasion & & 0.579 & \\
\hline Absent & $81.38 \pm 8.41$ & & Reference \\
\hline Present & $53.20 \pm 9.16$ & & $1.32(0.50-3.48)$ \\
\hline LN ratio & & $<0.001$ & \\
\hline$\leq 0.64$ & $105.67 \pm 5.11$ & & Reference \\
\hline$>0.64$ & $57.06 \pm 9.28$ & & $17.11(2.28-128.23)$ \\
\hline Metastatic LN number & & 0.001 & \\
\hline$\leq 15$ & $94.99 \pm 7.49$ & & Reference \\
\hline$>15$ & $55.84 \pm 10.77$ & & $4.75(1.70-13.23)$ \\
\hline
\end{tabular}

DFS, disease-free survival; $\mathrm{Cl}$, confidence interval; $\mathrm{HR}$, hormone receptor; HER2, human epidermal growth factor receptor 2; LN, lymph node.

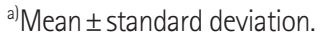


tively. A cutoff of 0.64 for LN ratio had a sensitivity of $95 \%$ and a specificity of $58 \%$, with LN ratio $>0.64$ being significantly associated with poor DFS (hazard ratio [HR], 26.13; 95\% CI, 3.16215.80). A cutoff of 15 for number of metastatic LNs had a sensitivity of $85 \%$ and a specificity of $58 \%$, with $>15$ metastatic LNs being significantly associated with poor DFS (HR, 4.60; 95\% CI, 1.38-15.32) (Fig. 1).

Based on these HRs, we constructed a scoring system to estimate the differences in survival of breast cancer patients with extensive axillary LN metastasis (Fig. 2). The score system included the tumor size, LN ratio and metastatic LN number, and ranged from 0 to 3 , with these subgroups consisting of $18,13,22$, and five patients respectively. Patients were dichotomized into two groups, those with scores of $0-1$ and 2-3. Five-year DFS rates in these two groups were $80 \%$ and $27 \%$, respectively, indicating that higher scores were associated with longer DFS.

\section{DISCUSSION}

Invasive breast cancer is the most common malignancy in women after thyroid cancer in Korea. Its most common sites of metastasis are the axillary LNs. Extensive LN involvement is associated with a significantly greater risk of poor prognosis, with LN status being the most important prognostic factor in breast cancer. LNs have been classified according to the American Joint Committee on Cancer LN-positive system, with stratification is based on the number of positive nodes ( $\mathrm{pN} 3$ is defined as $\geq 10$ positive nodes) [7].

Recent studies investigating the clinical outcome of breast cancer patients with pathologic N3 are summarized in Table $3[6,8$ 13]. More recent, studies have reported more improved outcome $70 \%$ to $87 \%$ of 5 -year OS rates and about 35\% to $72 \%$ DFS rates which was resulted by more advanced standard treatment. The present study found 5-year OS and DFS rates of 69\% and 55\%. However, these survival rates differed widely when patients were categorized by prognostic factors. None of the 18 patients with scores of 0 experienced local recurrence or distant metastasis, whereas these rates increased as the number of prognostic factors increased.

We confirmed that the number of metastatic LNs and LN ratio
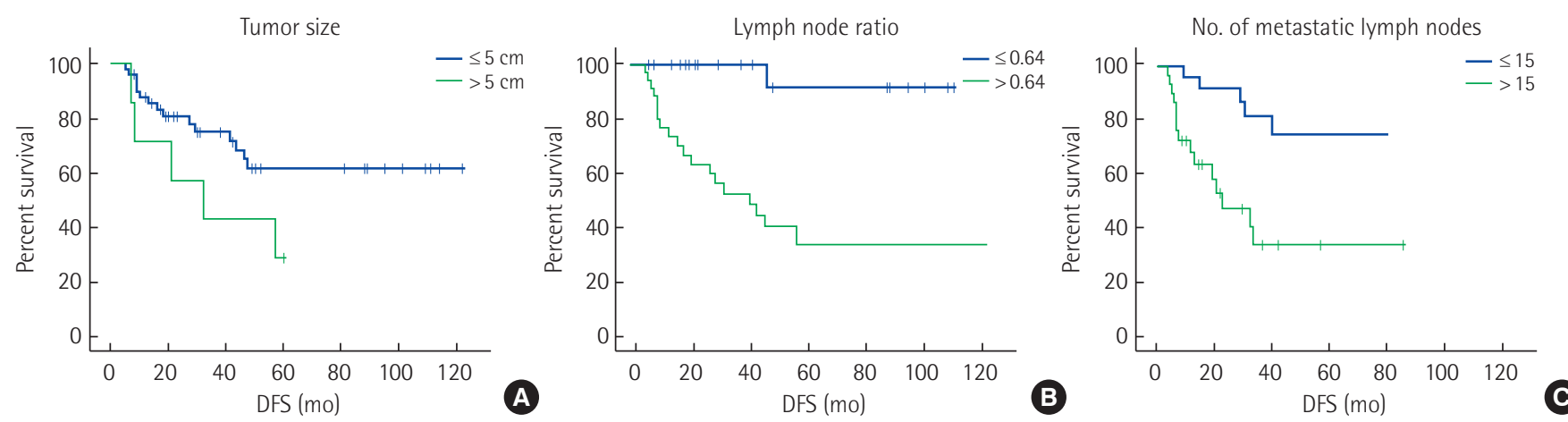

Fig. 1. Disease-free survival (DFS) curves according to prognostic factors. (A) Tumor size, (B) lymph node ratio and (C) number of metastatic lymph nodes were significant prognostic factors in DFS.

Scoring system

\begin{tabular}{|c|c|c|}
\hline \multicolumn{2}{|c|}{ Parameter } & \multirow{2}{*}{$\begin{array}{c}\text { Score } \\
0\end{array}$} \\
\hline Tumor size (cm) & $\leq 5$ & \\
\hline & $>5$ & 1 \\
\hline \multirow[t]{2}{*}{ Lymph node ratio } & $\leq 0.64$ & 0 \\
\hline & $>0.64$ & 1 \\
\hline \multirow[t]{2}{*}{ Metastatic lymph node numbers } & $\leq 15$ & 0 \\
\hline & $>15$ & 1 \\
\hline
\end{tabular}

\section{A}

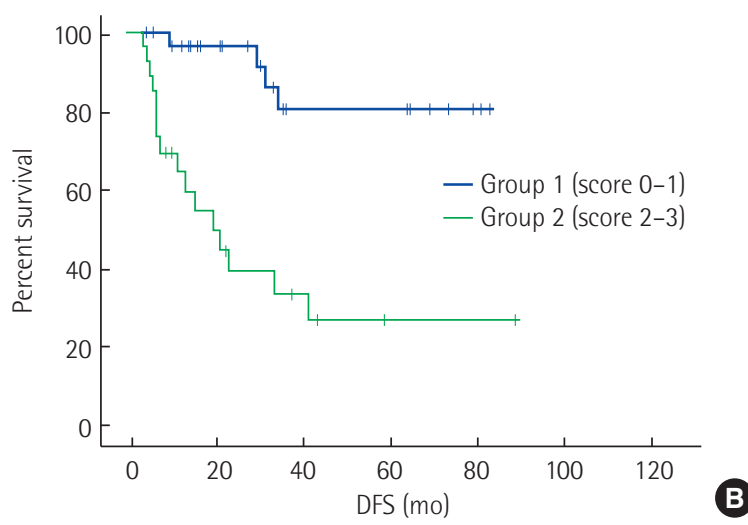

Fig. 2. Scoring system to predict the survival outcome. (A) Components of the scoring system and (B) survival difference according to subgroups. DFS, disease-free survival. 
Table 3. Review of recent studies about clinical outcome of N3 breast cancer patients

\begin{tabular}{|c|c|c|c|c|c|c|}
\hline Author & Year & Inclusion criteria & No. of patients & 5-Year DFS & 5-Year OS & Prognostic factor for DFS \\
\hline Duraker et al. [8] & 2008 & pN3a only & 313 & $35 \%$ & - & $\begin{array}{l}\text { Metastatic LN number }(>15) \\
\text { Nodal ratio }(>0.8)\end{array}$ \\
\hline Basaran et al. [6] & 2011 & pN3a only & 73 & $66 \%$ & $81 \%$ & $\begin{array}{l}\text { HER2 status } \\
\text { Metastatic LN number }(\geq 20)\end{array}$ \\
\hline Koca et al. [9] & 2013 & pN3a only & 218 & $46.2 \%$ & $69.8 \%$ & $\begin{array}{l}\text { Grade III } \\
\text { Perineural invasion } \\
\text { LN ratio ( } \geq 0.9)\end{array}$ \\
\hline Yu et al. [10] & 2015 & pN3 & 501 & $63.3 \%$ & $86.9 \%$ & $\begin{array}{l}\text { Age ( } \leq 40 \text { yr) } \\
\text { LN ratio (>60\%) } \\
\text { Nuclear grade (grade } 3 \text { ) } \\
\text { Molecular subtype }\end{array}$ \\
\hline Braunstein et al. [11] & 2015 & pN3a only & 98 & - & $71.9 \%$ & $\begin{array}{l}\text { Grade } \\
\text { Metastatic LNs ( } \geq 15)\end{array}$ \\
\hline Kim et al. [12] & 2016 & PN3 & 220 & $72.2 \%$ & $86.1 \%$ & $\begin{array}{l}\text { Age }(<35 \mathrm{yr}) \\
\text { T stage }(\mathrm{T} 3-4) \\
\text { LN ratio }(\leq 0.65) \\
\text { Neutrophil to lymphocyte ratio }(\leq 3) \\
\text { Tumor subtype }\end{array}$ \\
\hline Grassadonia et al. [13] & 2017 & pN3a only & 130 & $61.8 \%$ & $71.5 \%$ & $\begin{array}{l}\text { LN ratio (>60\%) } \\
\text { Neoadjuvant chemotherapy } \\
\text { Progesterone receptor } \\
\text { Molecular subtype }\end{array}$ \\
\hline
\end{tabular}

DFS, disease-free survival; OS, overall survival; LN, lymph node; HER2, human epidermal growth factor receptor 2.

were significant prognostic factors in patients with extensive regional LN involvement. Many studies of patients with node positive breast cancer have reported that LN ratio was prognostic, depending on the selected cut-off ratios. Two studies divided nodal ratio into low $(<0.2)$, intermediate $(0.2-0.65)$, and high $(>0.65)$ risk categories, and found that prognosis progressively worsened as nodal ratio increased $[14,15]$. Although these findings indicated that nodal ratio was an indicator of survival in patients with node-positive breast cancer, groups of patients with N1, N2 and N3 disease were not analyzed separately. In two studies composed of patient with pathologic N3, Duraker et al. [8] determined that the curoff value for nodal ratio is 0.8 while Kim et al. [12] found it to be 0.65 , and both studies showed that patients with a nodal ratio less than these values had better prognosis.

Molecular subtype is concerned the significant prognostic factor in breast cancer. However the results in N3 breast cancer showed the diverse results $[10,12,13]$. Yu et al. [10] reported that molecular subtype was associated with DFS only in univariate analysis not in multivariate analysis. In that study, although the patients with human epidermal growth factor receptor 2 (HER2) enrich breast cancer showed the most excellent prognosis, the patients with triple-negative breast cancer showed the poorest prognosis. The other study also that the patients with HER2 enrich and triple negative breast cancer showed poorer survival than the patient with luminal A breast cancer, and this result was statistically significant [13]. Although our study did not show a significant difference according to molecular subtype in DFS, the triple negative subtype tended to show the poorer survival outcomes than patients with other subtypes.

This study had several limitations, including its retrospective nature, which may have introduced a selection bias. Although we excluded patients who refused standard adjuvant chemotherapy, only about $33 \%$ of patients overexpressing HER2 received anti HER2 therapy. Moreover, the number of included patients was relatively small, and the median follow-up duration of 46.3 months was not sufficient to assess factors associated with long-term prognosis.

In conclusion, this study showed that survival outcomes in breast cancer patients with extensive LN metastasis were dependent on prognostic factors. Using our scoring system, we found that 5-year DFS rates were about 3-fold higher in patients with favorable than unfavorable factors. Management modification may improve clinical outcomes in patients with unfavorable factors. Additional large prospective studies, with longer-term follow-up, are necessary to confirm our results. 


\section{CONFLICT OF INTEREST}

No potential conflict of interest relevant to this article was reported.

\section{REFERENCES}

1. Kuru B, Camlibel M, Dinc S, Gulcelik MA, Alagol H. Prognostic significance of axillary node and infraclavicular lymph node status after mastectomy. Eur J Surg Oncol 2003;29:839-44.

2. Newman LA. Epidemiology of locally advanced breast cancer. Semin Radiat Oncol 2009;19:195-203.

3. DeSantis C, Siegel R, Bandi P, Jemal A. Breast cancer statistics, 2011. CA Cancer J Clin 2011;61:409-18.

4. Montero AJ, Rouzier R, Lluch A, Theriault RL, Buzdar AU, Delaloge S, et al. The natural history of breast carcinoma in patients with $>$ or $=10$ metastatic axillary lymph nodes before and after the advent of adjuvant therapy: a multiinstitutional retrospective study. Cancer 2005;104:229-35.

5. Jones SE, Moon TE, Bonadonna G, Valagussa P, Rivkin S, Buzdar A, et al. Comparison of different trials of adjuvant chemotherapy in stage II breast cancer using a natural history data base. Am J Clin Oncol 1987;10:387-95.

6. Basaran G, Devrim C, Caglar HB, Gulluoglu B, Kaya H, Seber S, et al. Clinical outcome of breast cancer patients with $\mathrm{N3a}(\geq 10$ positive lymph nodes) disease: has it changed over years? Med Oncol 2011;28:726-32.

7. Edge SB, Compton CC. The American Joint Committee on Cancer: the 7th edition of the AJCC cancer staging manual and the future of TNM. Ann Surg Oncol 2010;17:1471-4.
8. Duraker N, Caynak ZC, Bati B. Is there any prognostically different subgroup among patients with stage IIIC (any TN3M0) breast carcinoma? Ann Surg Oncol 2008;15:430-7.

9. Koca E, Kuzan TY, Dizdar O, Babacan T, Sahin I, Ararat E, et al. Outcomes of locally advanced breast cancer patients with $\geq 10$ positive axillary lymph nodes. Med Oncol 2013;30:615.

10. Yu JI, Park W, Choi DH, Huh SJ, Nam SJ, Kim SW, et al. Clinical outcomes and prognostic factors of pathologic $\mathrm{N} 3$ breast cancer treated with modern standard treatments. Clin Breast Cancer 2015;15:512-8.

11. Braunstein LZ, Galland-Girodet S, Goldberg S, Warren LE, Sadek BT, Shenouda MN, et al. Long-term outcomes among breast cancer patients with extensive regional lymph node involvement: implications for locoregional management. Breast Cancer Res Treat 2015;154:633-9.

12. Kim YY, Park HK, Lee KH, Kim KI, Chun YS. Prognostically distinctive subgroup in pathologic $\mathrm{N} 3$ breast cancer. J Breast Cancer 2016;19:163-8

13. Grassadonia A, Vici P, Gamucci T, Moscetti L, Pizzuti L, Mentuccia L, et al. Long-term outcome of breast cancer patients with pathologic N3a lymph node stage. Breast 2017;32:79-86.

14. Vinh-Hung V, Verkooijen HM, Fioretta G, Neyroud-Caspar I, Rapiti E, Vlastos $\mathrm{G}$, et al. Lymph node ratio as an alternative to $\mathrm{pN}$ staging in node-positive breast cancer. J Clin Oncol 2009;27:10628.

15. Liao GS, Chou YC, Golshan M, Hsu HM, Hong ZJ, Yu JC, et al. Prognostic value of the lymph node ratio in breast cancer subtypes. Am J Surg 2015;210:749-54. 